\title{
Disentangling Variability in Riverbank Macrolitter Observations
}

\section{Journal Article}

\section{Author(s):}

Roebroek, Caspar T.J.; Hut, Rolf; Vriend, Paul; De Winter, Winnie; Boonstra, Marijke; Van Emmerik, Tim H.M.

Publication date:

2021-04-20

Permanent link:

https://doi.org/10.3929/ethz-b-000487419

\section{Rights / license:}

Creative Commons Attribution-NonCommercial-NoDerivatives 4.0 International

\section{Originally published in:}

Environmental Science \& Technology 55(8), https://doi.org/10.1021/acs.est.0c08094 


\title{
Disentangling Variability in Riverbank Macrolitter Observations
}

\author{
Caspar T. J. Roebroek,* Rolf Hut, Paul Vriend, Winnie de Winter, Marijke Boonstra, \\ and Tim H. M. van Emmerik
}

Cite This: Environ. Sci. Technol. 2021, 55, 4932-4942

Read Online

ACCESS | Lلll Metrics \& More | 回 Article Recommendations | st Supporting Information

ABSTRACT: Anthropogenic macrolitter $(>0.5 \mathrm{~cm})$ in rivers is of increasing concern. It has been found to have an adverse effect on riverine ecosystem health, and the livelihoods of the communities depending on and living next to these ecosystems. Yet, little is known on how macrolitter reaches and propagates through these ecosystems. A better understanding of macrolitter transport dynamics is key in developing effective reduction, preventive, and cleanup measures. In this study, we analyzed a novel dataset of citizen science riverbank macrolitter observations in the Dutch Rhine-Meuse delta, spanning two years of observations on over 200 unique locations, with the litter categorized into 111 item categories according to the river-OSPAR protocol. With the use of regression models, we analyzed how much of the variation in the observations can be explained by hydrometeorology, observer bias, and location, and how much can instead be explained by temporal trends and seasonality. The results show that observation bias is very low, with only

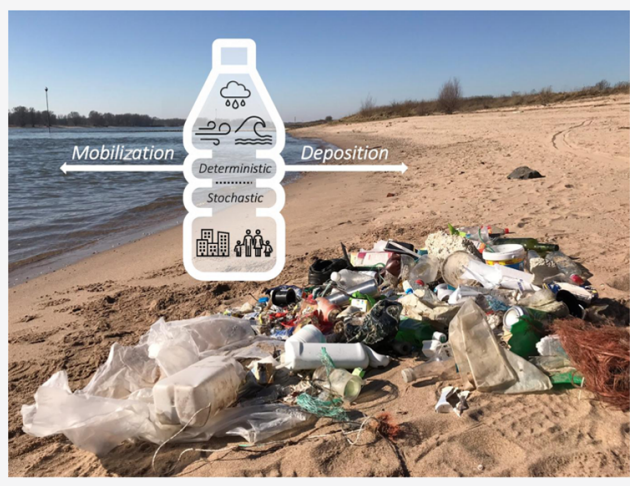
a few exceptions, in contrast with the total variance in the observations. Additionally, the models show that precipitation, wind speed, and river flow are all important explanatory variables in litter abundance variability. However, the total number of items that can significantly be explained by the regression models is $19 \%$ and only six item categories display an $\mathrm{R}^{2}$ above 0.4 . This suggests that a very substantial part of the variability in macrolitter abundance is a product of chance, caused by unaccounted (and often fundamentally unknowable) stochastic processes, rather than being driven by the deterministic processes studied in our analyses. The implications of these findings are that for modeling macrolitter movement through rivers effectively, a probabilistic approach and a strong uncertainty analysis are fundamental. In turn, point observations of macrolitter need to be planned to capture shortterm variability.

KEYWORDS: river-OSPAR protocol

\section{INTRODUCTION}

Anthropogenic macrolitter $(>0.5 \mathrm{~cm})$ in rivers is of increasing global concern. Depending on the material type and size, macrolitter negatively affects environmental health and human livelihood within and close to riverine ecosystems. ${ }^{1}$ Direct impacts of plastics include economic losses through damage to vessels, ${ }^{1,2}$ increased (urban) flood risk by blocking hydraulic infrastructure, ${ }^{3}$ and (fatal) damage to aquatic flora and fauna. ${ }^{1}$ With the predicted increase in the quantity of litter reaching the river systems, ${ }^{4,5}$ it becomes increasingly important to understand the source, mobilization, transport, and fate of the litter items.

Macrolitter arrives in the river network from a variety of sources ranging from industry, sewage overflows, and items that are directly disposed close to or in the river. Global data show that most of this litter is made of plastic but other materials such as wood, metal, glass, and paper are also found (e.g., Castro-Jiménez et al., 2019; van Emmerik et al., 2020). In the river system, the litter is distributed over several compartments. ${ }^{1}$ Inside the water itself, items can be separated into floating elements and items in suspension. From here, litter can be (temporarily) deposited on the riverbed, within the sediment, on riverbanks, in vegetation along and within the river, and sometimes even within the riverine biota, ${ }^{8}$ leading to a large variation in retention times. ${ }^{9-13}$ The natural transportation toward and through the river system is traditionally attributed to three hydrometeorological processes: transport by precipitation-driven surface runoff, wind, and river flow dynamics (as well as tidal dynamics). These processes are assumed to be key factors in explaining both spatial and temporal variability in riverine litter abundance ${ }^{14-16}$ although true quantification is still missing.

Many strategies have been proposed to study the presence and movement of macrolitter in the river systems. Most of the studies mainly focus on litter floating on the river surface or deposited on the riverbanks. Both methods yield inherently

Received: December 21, 2020

Revised: March 18, 2021

Accepted: March 18, 2021

Published: April 1, 2021

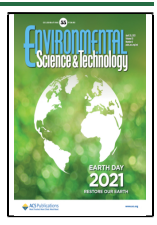


different information and can only be used as proxies to study the actual riverine litter transport. Floating litter observations are instantaneous measurements and only contain the buoyant fraction of the litter, while riverbank litter observations represent longer periods and contain a certain bias, as some items do not come from and will likely never reach the river (e.g., very heavy items that are abandoned on the riverbanks). Floating litter monitoring is often done by visual counting of the objects, ${ }^{17,18}$ both limiting the spatial distribution of the observations (observations are often done from bridges) and preventing detailed item descriptions and classifications to be made. Alternatively, the sampling can be performed through net sampling, after which the items can be weighed and classified. ${ }^{19}$ Although this method provides a more detailed description of the litter, it only provides a small snapshot in both space and time and is labor-intensive. Riverbank litter sampling provides the opportunity of obtaining a detailed description of the litter with high spatial resolution. ${ }^{20}$ Yet, few studies have explored the use of high detailed riverbank litter data to study the spatial and temporal variability of riverine litter. $^{18,21}$

In this study, we aim to make a first step in exploring the commonly assumed link between hydrometeorological processes and riverine litter transport by separating spatial and temporal variability in riverine macrolitter into observational bias, mechanistical processes, and a remaining stochastic component (which can be mainly attributed to human behavior). To do this, we make use of a dataset comprising over 150,000 sampled riverbank macrolitter items, obtained during a monitoring campaign along the Dutch Rhine-Meuse delta. In contrast with other monitoring campaigns (e.g., Kiessling et al., 2019; Owens \& Kamil, 2020), this covers a longer time period ( 2 years), has a high spatial coverage, uses the most detailed item categorization scheme to date (derived from the OSPAR categorization used for marine beach litter observations), and has built-in quality control as reference observations are performed in combination with citizen science (which is often solely used for such large-scale data retrieval). ${ }^{20}$ In previous studies where such data were analyzed, variability has been accounted for by determining hotspots in either space or time, which inherently cannot accommodate different sources of variability and is dependent on custom thresholds. Here, we use a combination of regression modeling and uncertainty analysis to determine the hydrometeorological processes that best explain the data, at item category level, creating highly targeted hypotheses on how single items reach and leave the riverbank. With the same approach, we express the explanatory power of time, seasonality, space, and type of observer (experts versus citizen scientists) to identify the additional sources of variability in the observations. Ultimately, combining these factors together in a single regression model, the variance in the data is attributed to deterministic processes accounted for in this study and stochastic events capturing unaccounted processes.

\section{METHODOLOGY}

Litter Dataset. The litter observation data used in this study was obtained via a monitoring campaign conducted by The North Sea Foundation (Dutch: Stichting De Noordzee), henceforth referred to as SDN. In this campaign, riverbanks along the Dutch Meuse and Waal (branch of the Rhine) were observed between 2017 and 2019. In total, 384 observations were carried out in four rounds: autumn 2017, spring 2018, autumn 2018, and spring 2019, with each round lasting four weeks. In each round, a pair of volunteers were assigned a specific, predefined location (day of the measurement was chosen by the volunteer, within the four-week window). During each observation, all macrolitter $(>2.5 \mathrm{~cm})$ was removed from a $100 \mathrm{~m}$ riverbank stretch and categorized according to the River-OSPAR protocol, ${ }^{7}$ containing 111 unique item categories (see Figure 2 for a complete list). The microlitter items (between 0.5 and $2.5 \mathrm{~cm}$ ) were separately obtained and classified by randomly selecting a quadrant of 50 $\mathrm{cm} \times 50 \mathrm{~cm}$ on the riverbank and extrapolating the number of items to the whole area (for details, see Van Emmerik et al. (2020)). Blanks in the dataset can, therefore, be interpreted as zero items of this category being present on the riverbank. The width of the sampling area was determined by the characteristics of the riverbank and is defined as the distance from the water line to the high water line during the latest peak discharge, with a maximum distance of 25 meters. Each location was at most sampled once per measurement round. The observations, therefore, represent the accumulation that took place between samples, integrating litter input and output, including undocumented removal. In total, 152415 items were sampled at 212 unique locations. Most locations were observed at least twice, and some locations were observed during every measurement round. The target area for each observation was $2500 \mathrm{~m}^{2}$ (100 meters along the river and 25 meters perpendicular to the river), but not all observations fit this criterion. In this study, we normalized the data by linearly extrapolating the observed litter density to the target $2500 \mathrm{~m}^{2}$ (for example, if only $1000 \mathrm{~m}^{2}$ were sampled, the observations were multiplied by 2.5 ). To get an estimate of the quality and accuracy of the data gathered by the volunteers, reference observations were carried out by SDN staff (a total of 21 observations, spread over the four measurement rounds). Due to the novelty, no standardized quality control protocol has been developed to date.

Additional Data. To be able to study the influence of hydrometeorology on litter distribution, we combined the publicly available data on precipitation and wind speed (20172019) from 50 measurement stations of the Royal Netherlands Meteorological Institute (KNMI) and water level from 87 measurement stations of the Netherlands Ministry of Infrastructure and Water Management (Rijkswaterstaat) with the observational litter data. In line with the common assumption that most litter (with sizes ranging from woody debris to microplastics) is mobilized and transported during hydrometeorological maxima, ${ }^{10,22,23}$ we calculated the maximum precipitation, wind speed, and water level over several time intervals prior to each single litter observation ( 2 days, 7 days, 14 days, 1 month, 2 months, and 6 months). The values at each litter observational site were estimated by interpolating the data (from national hydrometeorological measurement stations) using inverse distance weighting (one-dimensional (1D) for water level and two-dimensional (2D) for precipitation and wind speed).

Besides the trends along the hydrometeorological gradient, we analyzed the litter data on trends in time and categorically on seasonality (spring vs autumn, respectively, 285 vs 99 observations) for differences between rivers (Meuse vs Waal, respectively, 246 and 138 observations) and sampling strategy (volunteer sampling vs reference sampling by experts, respectively, 363 and 21 observations). The variables with a short description and their units are presented in Table 1. 
Table 1. Variables Used in the Regression Models Including Their Units and Description

\begin{tabular}{|c|c|c|c|}
\hline variable & unit & categorical & description \\
\hline precipitation & {$\left[\mathrm{mm} \mathrm{d}^{-1}\right]$} & no & maximum daily precipitation sum in the optimal time interval preceding the litter observation \\
\hline wind speed & {$\left[\mathrm{m} \mathrm{s}^{-1}\right]$} & no & maximum daily mean wind speed in the optimal time interval preceding the litter observation \\
\hline water level & {$[\mathrm{m}]$} & no & $\begin{array}{l}\text { maximum water level above the level during the observation in the optimal time interval preceding the litter } \\
\text { observation }\end{array}$ \\
\hline time & {$[\mathrm{d}]$} & no & number of days since January 1, 2017 \\
\hline time after last observation & {$[\mathrm{d}]$} & no & number of days since the last observation at the same site (if any) \\
\hline $\begin{array}{l}\text { seasonality (spring vs } \\
\text { autumn) }\end{array}$ & {$[-]$} & yes & number of observed items during a spring measurement in comparison with an autumn measurement \\
\hline Waal vs Meuse & {$[-]$} & yes & number of observed items along the Waal in comparison with the Meuse \\
\hline volunteer vs reference & {$[-]$} & yes & number of observed items by volunteers in comparison with reference observations \\
\hline
\end{tabular}

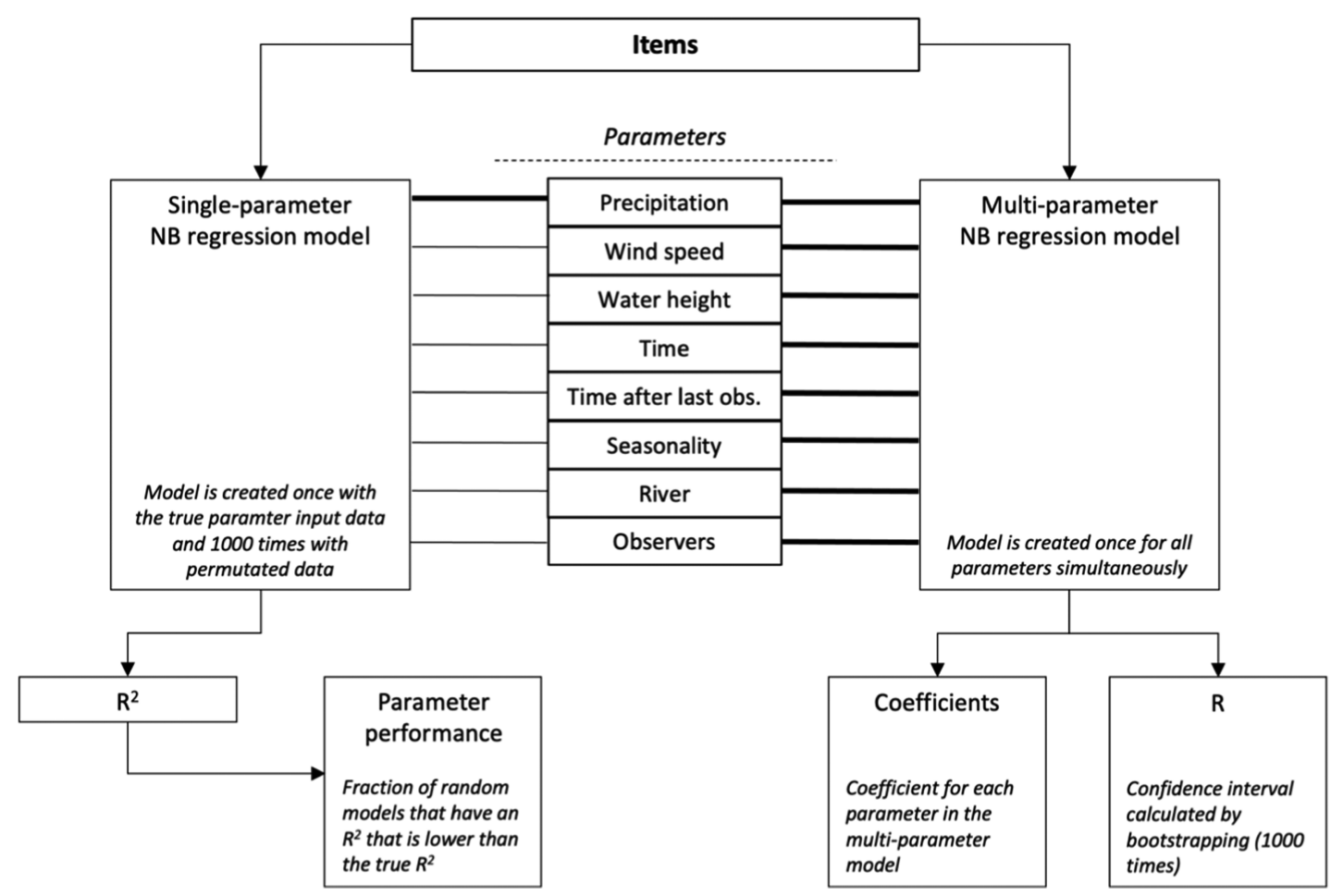

Figure 1. Flowchart describing the different components of the data analysis. The thick black lines from parameters to the components describe the input to the models. All parameters are simultaneously included in the multiparameter models, while the single-parameter models are created for each individual parameter.

Statistical Model. To best attribute the effects of these parameters on the observed litter quantities, we used negative binomial linear regression models for each parameter and item category combination. Negative binomial regression is a generalization of Poisson regression, often used in ecology to study population count data. It is based on the Poisson- $\gamma$ mixture distribution, and used here as litter observations are count data, often displaying overdispersion (higher variance than mean) thus ruling out direct Poisson regression. ${ }^{24,25}$ This type of model expresses the item count $(C)$ as drawn from a negative binomial distribution, with parameters $r$ (predefined number of successes in a Bernoulli trial) and $p$ (probability of a success of these trials).

$$
C \sim N B(r, p)
$$

The model predictions (mean $\mu_{i}$, for each item) are defined such that the natural logarithm of the prediction follows linearly from a linear combination of the predictor variables. Here $X$ is the vector describing the predictor variables, $\beta_{i, j}$ is the parameter for item $i$ and predictor variable $j$, and $\alpha$ is the intercept. $M$ indicates the number of predictor variables.

$$
\begin{aligned}
& E(C)=\mu_{i} \\
& \ln \left(\mu_{i}\right)=a_{i}+\beta_{i} X_{i}^{T} \\
& \beta_{i}=\left[\begin{array}{c}
\beta_{i, 1} \\
\vdots \\
\beta_{i, M}
\end{array}\right]
\end{aligned}
$$

Zero-inflated negative binomial (ZINB) regression models were deemed unsuitable in this scenario, as zero count observations are part of the same stochastic and mechanistic processes determining the distribution of the litter.

Hydrometeorological fluxes (precipitation, wind speed, and river discharge) transport litter only when exceeding a certain threshold (stemming from physical laws of motion). Therefore, each item-flux pair has a typical timescale on which the transport occurs. To assess these timescales, regression models 


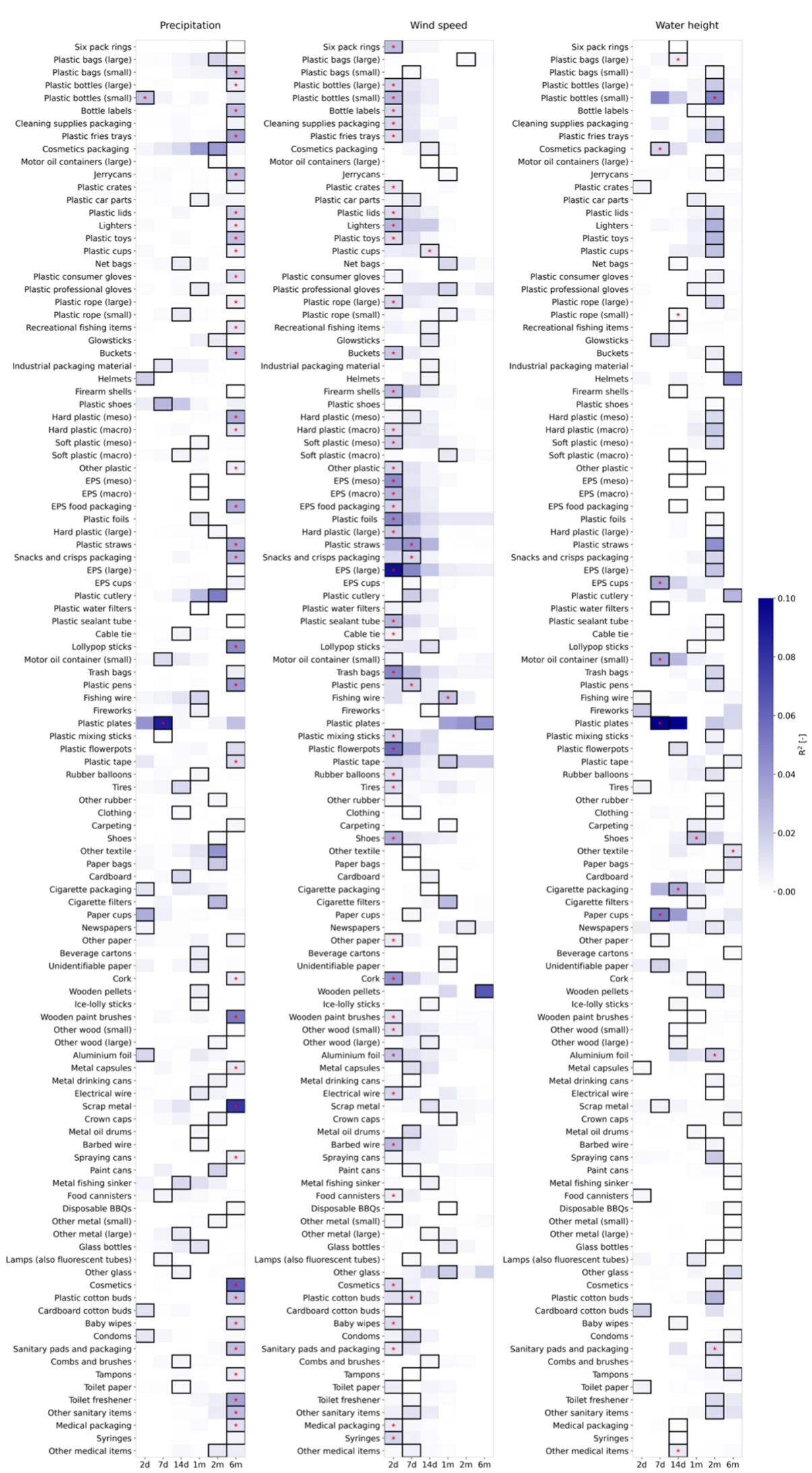

Figure 2. Optimal time interval for each precipitation-, wind speed-, and water height-item pair, calculated by generating a regression model between each item and all hydrometeorological fluxes over the different time spans. The $x$-axis of the figure shows the time lags $(2$ days, 7 days, 14 days, 1 month, 2 months, and 6 months). The colors in the figure correspond to the $R^{2}$ value of the regression model, and the optimal lag per item is highlighted with a black box. The significance ( 90 percent of the random models performing worse than the actual model) is shown with a red star (note that they are only shown for the optimal time intervals). 

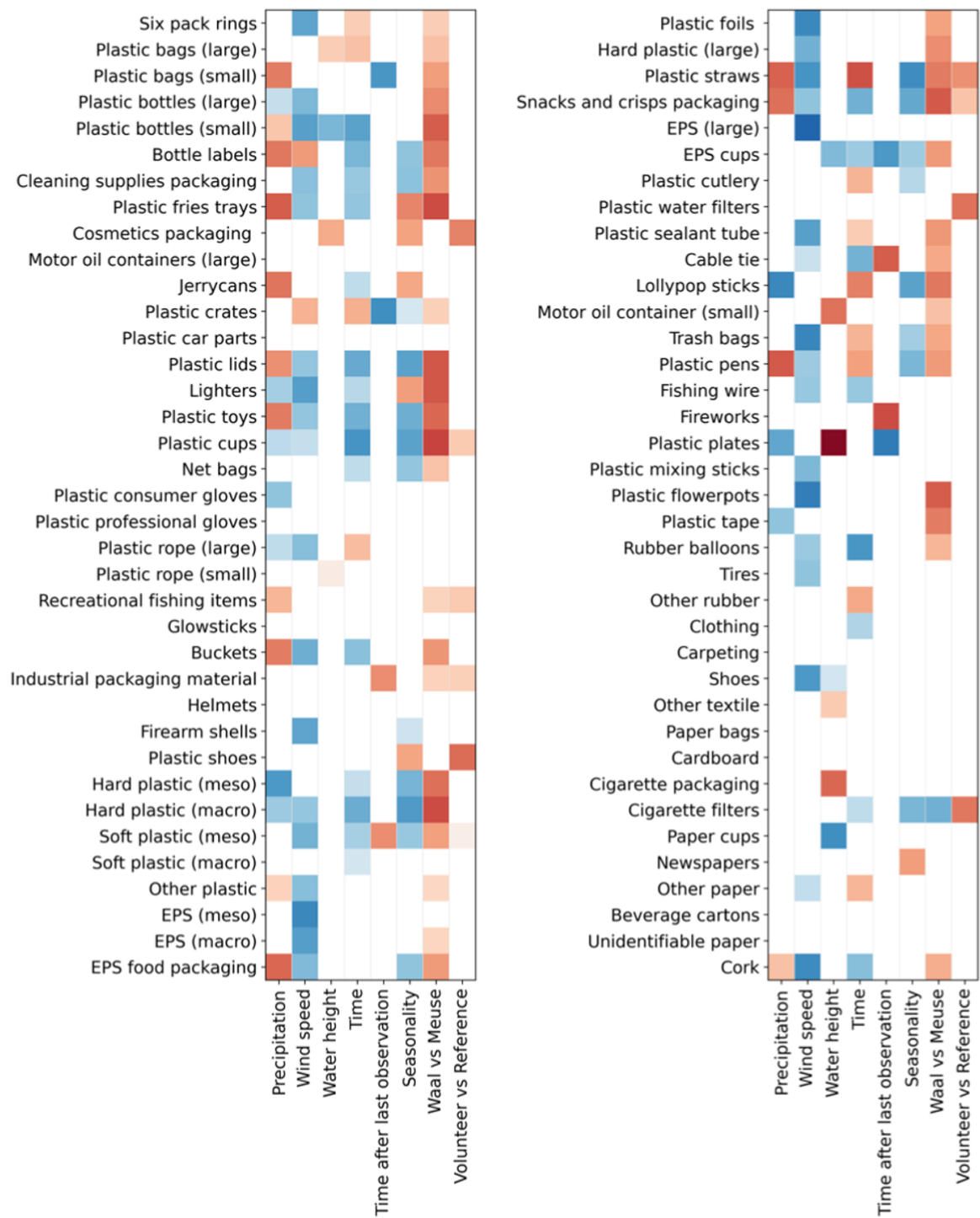

Wooden pellets Ice-lolly sticks Wooden paint brushes Other wood (small) Other wood (large)

Aluminium foil

Metal capsules Metal drinking cans Electrical wire Scrap metal Crown caps Metal oil drums Barbed wire

Spraying cans Paint cans Metal fishing sinker Food cannisters Disposable BBQs Other metal (small) Other metal (large) Glass bottles Lamps (also fluorescent tubes) Other glass Cosmetics Plastic cotton buds Cardboard cotton buds Baby wipes Condoms

Sanitary pads and packaging Combs and brushes Tampons Toilet paper Toilet freshener Other sanitary items Medical packaging Syringes Other medical items

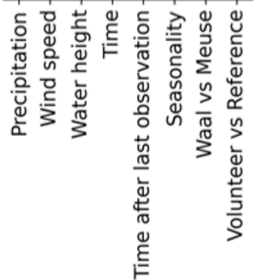

\begin{tabular}{lllllll}
\hline-0.2 & -0.1 & -0.01 & 0 & 0.01 & 0.1 & 0.2
\end{tabular}

Figure 3. Representation of explanatory power and sign of precipitation, wind speed, and water level as well as time, seasonality, river, and observer type on the different items. Only combinations presented in blue (positive correlation) or red (negative) were found significant. This figure can be interpreted as follows: [water height - plastic plates] shows a negative relation. Therefore, if the water level was much higher in the weeks preceding the riverbank observation (than during the observation), the chance of finding plastic plates is substantially lower.

are set up, modeling each item separately as a function of the maximum hydrometeorological flux over several time spans (as described above). The time span yielding the best model performance (highest $R^{2}$ ) is used in the analyses described below.

To assess the individual influence of the hydrometeorological fluxes, time, seasonality, location, and sampling strategy, regression models are created for each parameter-item combination. As the presence of specific litter items in many cases can be attributed to single events (such as visitors leaving litter behind at one specific riverbank), we performed a permutation test for uncertainty analysis. To achieve this, the parameter-item models were recalculated 1000 times while randomly shuffling the parameter data. In this study, we used a $90 \%$ threshold of the $R^{2}$ of the true model exceeding the $R^{2}$ of the random models to separate the true effect of the parameter on the litter distribution from the stochasticity in the presence of specific litter items. In the text, we refer to these models as the single-parameter models (see Figure 1).

The same regression models including all parameters per item category were separately set up (here referred to as multiparameter models). This was done to determine the coefficients per parameter-item pair (rather than using the coefficients form the single-parameter models, to compensate for overlapping effects). Additionally, the Pearson correlation $(R)$ between the observed and modeled item counts was calculated to assess how much of the variation in the input data can be explained by hydrometeorology, trends in time and space, and by the observation strategy (volunteer vs reference). Finally, these correlation values are analyzed on uncertainty by performing a 1000-time bootstrap (correlation value of a sample, with replacement, of the observed/modeled item 
count pair). This yields a normal distribution around the true correlation value. It was assumed that the explanatory power of the model is significant if the lower $99 \%$ confidence boundary yields a positive correlation value. In other words, if the correlation value is equal to or higher than 2.3 times the standard deviation of the correlation value (in a one-tailed approach) the model is deemed significant. Finally, a percentage of variance that can be addressed with this modeling approach is calculated by multiplying the $\mathrm{R}^{2}$ of the models that significantly explain an item with the number of observations of the item, summing up these values, and dividing it by the total number of items included in the database. More details can be found in the Supporting Information.

\section{RESULTS AND DISCUSSION}

Timescale of Transport. The results show that 90 of the 111 item categories are significantly explained by at least one of the included parameters, while 72 items show a significant correlation between hydrometeorology and item abundance (Figures 2 and 3). The optimal time span between the hydrometeorological flux-item pairs shows that precipitation, wind speed, and discharge (approximated by water height) operate on very different timescales. Most significant models predicting item abundance from wind speed are optimal on the scale of days, most models from water height on the scale of weeks, while a majority of the models significantly predicting item abundance from precipitation show an optimal time lag of months. The significant relations (and their sign) are shown in Figure 3 and are subsequently explored in the following paragraphs, leading to the formulation of several hypotheses on the modes of transport of litter through the environment.

Variables. Precipitation. Runoff, and with that precipitation, has been hypothesized to be an important driver in litter transport. For example, the first global modeling efforts on predicting plastic transport toward the sea heavily rely on runoff estimates to derive riverine plastic loads. ${ }^{14}$ In contrast, we find that only a third of the items (see Figures 2 and 3) is significantly explained by precipitation. Even items that are predominantly present on riverbanks because they are left behind (such as cigarette packaging and paper cups) do not show negative relation between abundance and precipitation, which could be expected from the hypothesis that runoff is a major driver in litter transport. Runoff in built-up areas and extreme precipitation events do, however, likely play a significant role in litter transport (as reflected by the long optimal time lags of precipitation on item abundance in combination with the resulting negative coefficients). Additionally, it needs to be noted that a similar study in a country with a steeper terrain than the Netherlands might have resulted in stronger precipitation and item-abundance relationships. Nevertheless, the fact that a majority of the items in this data analysis are not significantly explained by precipitation-driven transport calls for caution in attributing litter transport to runoff (without differentiating in item characteristics).

An interesting hypothesis that can be drawn from the negative relationships between precipitation and litter abundance in combination with the long optimal time intervals is that during periods with even moderate precipitation less visitors to the riverbanks can be expected (e.g., winter season, to which we will come back later). An additional potential pathway of precipitation driving litter abundance are sewage overflows. During extreme precipitation events, sewers can overflow, potentially dumping litter items in the open water from where it can be deposited on the riverbanks. A possible example from this data analysis might be toilet freshener, showing a positive correlation with precipitation (with an optimal time interval of 6 months), as well as a positive relation with season (higher values found in spring in comparison to autumn, which hints at sewage overflow transport occurring during the winter months). These processes together describe a starkly nonlinear precipitation-litter relation, with the highest litter abundance being expected under either heavy rainfall or no rainfall at all. To resolve these transport mechanisms, data during extreme events and on riverbank residence times are needed. ${ }^{10,11}$

Wind Speed. Wind is shown here to be a more important mode of transport of litter items to the riverbanks than either runoff (precipitation) or river deposition (water level). Especially, plastic items show a substantial number of significant relations between wind speed and item count. This corresponds to the intuitive understanding that, especially, small and lightweight items are easily transported with high wind speeds. The nonplastic items that show a significant relation with wind speed are also relatively lightweight, such as aluminum foil and plastic cotton buds. An interesting exception on this is the item category tires, but as only relatively few have been observed, this relation can be seen as the product of chance. Other, more often observed heavy items such as disposable barbecues do not show a significant link with wind speed. Interestingly, paper items do also not show significant links, although they can likely be transported by wind. This might be explained by the fact that these items fall apart relatively quickly and might therefore not arrive on the riverbanks as recognizable items (reflected by the positive coefficient of the wind speed-other paper relationship). Alternatively (or additionally), the amount of paper moving away from and toward the riverbanks could be equal, resulting in the absence of a significant link between wind speed and paper items. Additionally, under high wind speeds, items within the river can potentially be deposited on the riverbanks, which might explain the fact that the smallest plastic items (e.g., soft meso plastic) are found to be positively related to wind speed (see Figure 3).

Water Level. Water level is defined here as the highest water level above the water level during the litter observation over the previously defined optimal time interval (see Figure 2). It, thus, describes the potential of direct deposition of items on the riverbanks as well as the potential of river water to clean the riverbanks. Relatively, few items show a significant relationship with water level, with most of these items being either plastic or paper. The explanation of these relationships depends on the material type. Many plastic items float on water and are therefore likely to be deposited on riverbanks during high water events. The presence of plastic items with higher density that do not float on water - or items that can contain water and therefore do not float such as jerrycans and plastic car parts - is not significantly explained by water level. Also, the smallest plastic items (hard plastic (meso) and soft plastic (meso)) are not explained by water level. This lack of correlation may be explained by two different processes: (1) either these items are not abundant enough in the river water with respect to the riverbanks to have a significant net flux toward the riverbanks, or (2) these small fragments do not actually deposit during high water events. In contrast, paper items do not float and are likely not deposited during high 

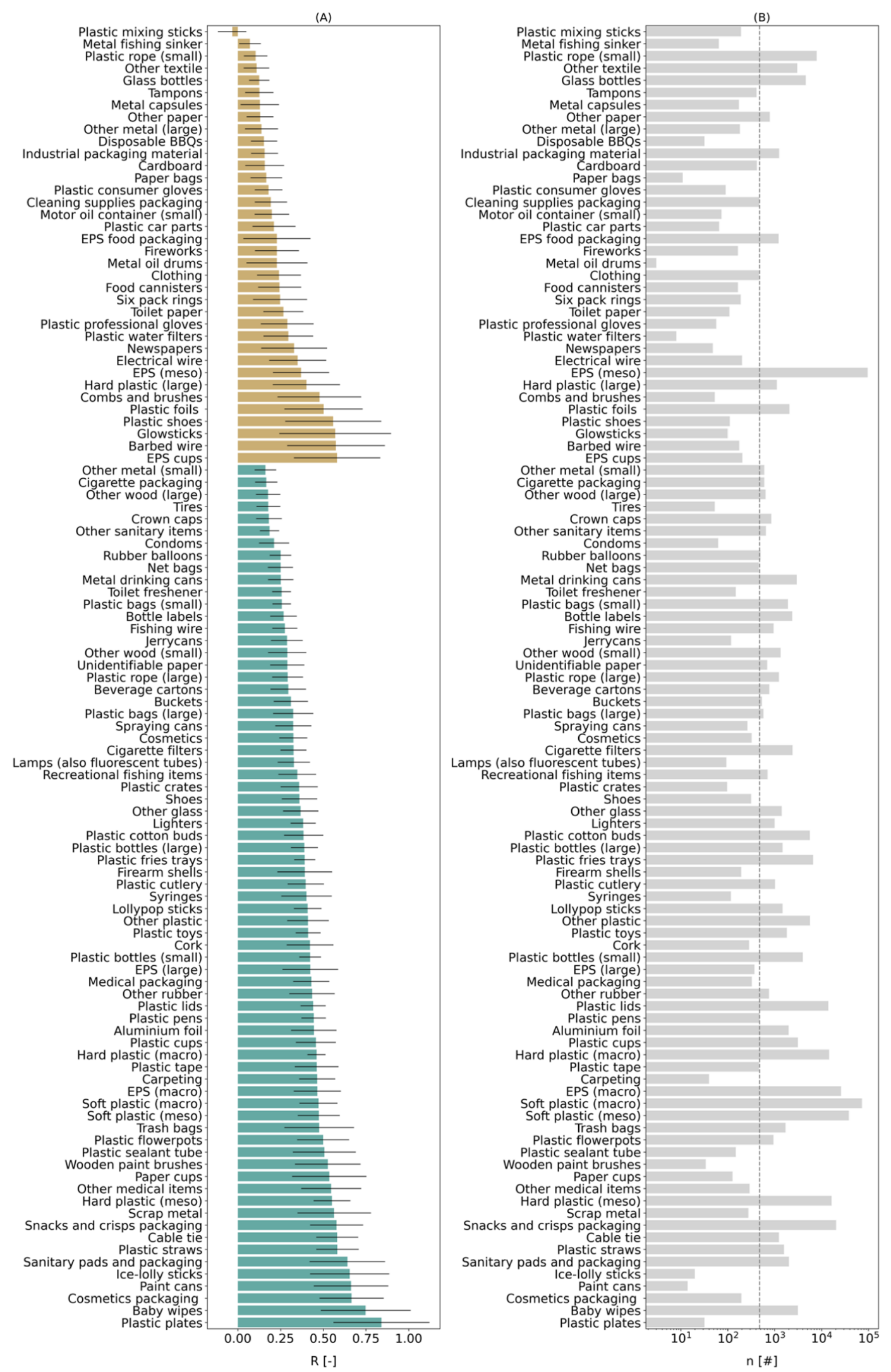

Figure 4. Illustration of the explanatory power of the multiparameter models on the abundance of the different items. (A) Correlation between observed and modeled item counts with the black lines representing two standard deviations around this value. The items displayed in brown are not significantly explained by the models, while the items in green are. (B) Number of these items that were observed. The median number of items is given by a dashed vertical line. 
water events. The positive relation between, for example, paper cups and water level is, therefore, more likely to be explained by the capacity of paper to absorb and store water. During high water events, paper items on the riverbanks get soaked and increase dramatically in mass, reducing their transportability by wind and runoff. As a result, the number of paper items found after high water events can be substantially higher. Indeed, for paper cups, the optimal time interval is one week, which reflects the drying and (re)transportation of such items. Note that the rational presented here only considers discharges that do not cause flooding. During flood events, a substantial amount of additional litter might be transported and, in turn, deposited on the riverbanks. ${ }^{23}$

Time. Many items show a significantly positive link between their abundance and time. Although the parameter values are relatively low, thus, only showing a slight increase in abundance, it is a worrying result of the data acquisition campaign. As the data are obtained in four measuring rounds spread over a period of two years, the trends in time are still relatively premature. More definitive analyses can only be made with longer time series. An interesting observation in this data is that plastic bags show a negative trend in time, possibly corresponding to a national ban on free plastic bags that came into action in 2016. Gradually, the number of plastic bags in circulation was expected to decline in the years following the ban. Interestingly, this finding agrees with similar observations on the North-Sea beaches. ${ }^{26}$ Additionally, the time after the last observation shows a significant relationship only for a handful of items. This can be explained with the idea that most items have a lower residence time on the riverbanks than the measurement frequency.

Seasonality. In contrast with the trends of item abundance over time, this parameter explains seasonal variation. It is defined categorically between spring and autumn (corresponding to two measurement rounds occurring in spring and two in autumn); a positive relation means that more items are found during spring. Most items show such a positive relationship. Especially, as the Waal (Rhine) has much higher discharges in winter than in summer, these trends can likely be explained by item deposition and accumulation in winter and spring, while in summer, the item abundance decreases as a result of item removal by visitors and/or wind. Other items, mostly related to tourisms such as newspapers and metal drinking cans, show a negative relationship, i.e., less items are found during the spring measurement round. This corresponds to littering during the summer by visitors and removal over the course of the winter by high water events or human waste removal.

Waal vs Meuse. To account for the location of the observations, the river was included as a categorical parameter. Most items are more abundant along the Meuse than the Waal. Although the Waal (Rhine) has a higher yearly discharge and discharge variability (see, for example, Van Emmerik et al., ${ }^{7}$ ) in comparison with the Meuse, the data on water level included here shows only a slight difference between the rivers in variability of water height over the defined time intervals before the litter observations (except for the six-month interval). Differences are, therefore, expected to coincide instead with the surroundings of the riverbanks; more cities and industrial areas are located along the Meuse (e.g., van Emmerik et al., 2020), likely causing this apparent disparity between the two rivers. Litter can find its way to the river system through many different pathways such as incorrect waste disposal by visitors, dumping/accidental loss from ships and fisheries, and sewage overflows. The different litter densities in Meuse and Waal might originate from differences in all of these drivers. Additionally, it needs to be considered that increased riverbank litter abundance was found in the border regions with Germany and Belgium, suggesting a (substantial) contribution from Germany (Waal) and Belgium (Meuse) to Dutch rivers. ${ }^{7}$

Volunteer vs Reference. The categorical parameter Volunteer vs Reference is included to reflect the campaign strategy to use citizen science to obtain the data and perform reference samples to check data consistency and accuracy. Most items do not show any significant bias of volunteers, showing the value of citizen science. Most of the items showing a significant link between sampling strategy and item abundance were not found often, making the case that these biases are likely driven by chance rather than observational bias. The items that stand out are soft plastic (meso) and the sanitary items. Both were found significantly less by volunteers than during the reference samples. The observation bias in small soft plastic fragments may be explained by low detectability, while the sanitary items, plastic cotton buds $(n=$ 5690), baby wipes $(n=3232)$, and sanitary pads and packaging $(n=2011)$, may instead be related to item aversion by volunteers. Note, however, that the coefficients in comparison with the average number found per observation signify that volunteers find on average 10 percent less of these three item categories.

Hydrometeorology as a Driving Force. The models used in this study represent the effects of hydrometeorology, time, location, and observational strategy on the abundance of the different item categories. By interpreting the correlation between the modeled and observed item counts, it becomes possible to separate the items into two categories: predictable and unpredictable items (see the last paragraph of Methodology). They are shown in Figure 4, displayed in green and brown colors, respectively. The unpredictable category contains items such as disposable barbecues, fireworks, and metal oil drums, which are likely left behind by visitors. This corresponds to the observation bias of riverbank observations with respect to riverine litter transport: some items are likely not transported by the river but are nonetheless present on the riverbanks. Items that are likely transported by the river such as soft plastic (meso), plastic bottles, and EPS parts (medium and large sizes of the smallest parts are represented as unpredictable) fall in the predictable category. From our analysis, we found that the fraction of the total amount of items that can be predicted through hydrometeorology and observation metadata is 15 percent. As item mass has not been observed in the measurement campaign, it is difficult to represent the fraction of mass that falls within the predictable category, but by removing the smallest and lightest items (categories containing the word meso) from the above analysis yields a fraction of 19 percent that can be classified as predictable.

\section{SYNTHESIS AND OUTLOOK}

In this study, we evaluate the data of riverbank macrolitter observations in the Dutch Meuse-Rhine delta obtained by SDN between autumn 2017 and spring 2019, using the RiverOSPAR protocol. The monitoring campaign was performed to quantify litter abundance at the national scale, find hotspots of litter along the rivers in the Netherlands, and provide itembased trends for policy and intervention purposes. As the data 
are extensive in both space and time and item categorization, however, it creates not only the potential of evaluating the monitoring campaign but also makes it possible to analyze how environmental factors, such as wind, precipitation, and river flow affect the number and composition of items found on riverbanks. The combination of these hydrometeorological factors together with the understanding of the physical properties of the different items creates the potential of stating the first data-driven hypotheses on litter transportation toward and along river systems. To do this, we used a statistical approach, linking hydrometeorological data and observation metadata to item counts with negative binomial regression models. Although the parameters for these models are far from exhaustive and many more could potentially be added, it gives an overview of the main mechanistical processes hypothesized to transport macrolitter.

The analysis presented here shows that trends in both space and time are significant for many items, from all parent item categories and from a large range of sizes. More items are consistently found along the Meuse than along the Waal, which is likely linked to a higher number of industrial areas and cities lying along the river. At the same time, almost all items significantly explained by time show a positive trend, hinting at a gradual increase of litter items on Dutch riverbanks over the observed years. Temporal variance in litter quantities is explained not only by trends in time. The data shows that a substantial number of item categories show a significant seasonal trend. This is linked to changing composition and source of the different litter items: in summer, riverbanks are used more intensively for recreational activities, and more visitor-related items are found during the fall measuring round. At the same time, the higher discharges in the winter partially remove these items while depositing mainly lighter and smaller items on the riverbanks. Moreover, the results suggest that the average residence items of most item categories is lower than the measurement frequency (6 months), shown in the lack of a substantial number of items positively correlated with the time between litter observations. Additionally, the results validate the monitoring campaign by comparing reference measurements with observations by volunteers. The results show that for most items no significant observer bias is present, with the exception of some very small objects and sanitary items that do indeed show a significant negative observation bias by volunteers, which can potentially be linked to item aversion.

Besides between-year and seasonal temporal trends, the results show that many items are significantly related to flow velocities, yielding an additional dimension in temporal variance in litter abundance. Global modeling studies, such as Lebreton et al. (2017), use precipitation-driven surface runoff and/or river discharge to explain (plastic) litter transport. In contrast, we find that items more often significantly correlate with wind speed than with either precipitation or river discharge. Furthermore, these transport mechanisms potentially operate on very different timescales. From the data, we find that wind speed explains item abundance best at the scale of days and discharge on the scale of weeks, while precipitation shows the strongest signals at the scale of months. Additionally, it is interesting to note that relatively similar items show quite different behaviors. For example, undefined pieces of EPS (meso, macro, large) only show a significant relation with wind speed, while EPS food packaging also shows a negative relationship with precipitation. On the other hand, EPS cups correlate positively to discharge, while not displaying any relation to wind speed or precipitation. This leads to the notion that studying litter transport in the environment with a detailed item characterization (which is here approximated by a categorization with the extensive River-OSPAR protocol) can lead to very different results than when binning on material/functional categories, which is quite common in macrolitter research. ${ }^{20}$ Items of similar material or with a similar function, but with different shapes and sizes, react quite differently to hydrometeorology. Additionally, it can be noted that items that can contain water, such as EPS and paper cups, often show a significant relation with water level. This can likely be better explained by fixation, inhibition of movement through increasing mass, rather than deposition by the river. Although the results show significant relationships between litter quantities and hydrometeorology, it needs to be considered that they cannot be interpreted as causal relationships. Instead, the results provide a base for more direct experimental studies that can link these processes to movement. These experimental studies might, for example, take place in the form of flume experiments to mimic river flow and wind tunnel tests to determine critical wind speeds for the transport of different items and materials. It might be an interesting start to use the abundant items used in this study (e.g., above the median item count as shown in Figure 4B) that were best predicted with the multiparameter regression models as displayed in Figure 4.

Although many items show a significant relation with wind speed, precipitation, or water level, the multiparameter models including both hydrometeorology and the monitoring metadata can only significantly explain the variation of 19 percent of the larger items $(>2.5 \mathrm{~cm})$. At the same time, only 6 of the 111 items show a final multiparameter-model $R^{2}$ value above 0.4. Note that the list of included variables is exhaustive. By including more variables (especially on vicinity to cities and industrial areas or the use of the riverbank for recreational purposes), some of the unexplained variance might be captured and the explained fraction might increase. Nevertheless, the results suggest that much of the variation in item counts is stochastic, i.e., single events (such as visitors coming to riverbanks) are an important contributor to riverbank litter. The observed, nondeterministic nature of both spatial and temporal variations of riverine litter leads to the consideration that both monitoring and modeling of the litter need to be adjusted accordingly. The most thorough, direct observations campaigns of riverine litter are done by repeatedly observing floating litter for a relatively short amount of time, which is subsequently extrapolated to hourly, daily, and even yearly values. ${ }^{6}$ This methodology implicitly uses the assumption that variability would be best explained by covariability with river flow. Following the logic explained in this work, the conclusions that are taken from such observations would need to be constrained by observing short-term variability (with similar river discharges) and comparing these values to the variability attributed to hydrometeorology. This can, for example, be achieved by observing the floating litter flux several times on a single day on relatively short intervals. Similarly, for riverine litter modeling, uncertainty due to nondeterministic variability needs to be considered as an important constraint, requiring strong uncertainty analysis and/or probabilistic modeling approaches. In turn, understanding the variability of (macro)litter in both space and time is an important step in designing effective policies, mitigation strategies, and cleanup actions. 


\section{ASSOCIATED CONTENT}

\section{(5) Supporting Information}

The Supporting Information is available free of charge at https://pubs.acs.org/doi/10.1021/acs.est.0c08094.

An extension on the methods section, with a more indepth explanation of the modeling choices (PDF)

\section{AUTHOR INFORMATION}

\section{Corresponding Author}

Caspar T. J. Roebroek - Hydrology and Quantitative Water Management Group, Wageningen University, 6708 PB Wageningen, The Netherlands; Institute for Atmospheric and Climate Science, ETH Zürich, 8092 Zürich, Switzerland; (-) orcid.org/0000-0002-1733-0845;

Email: caspar.roebroek@env.ethz.ch

\section{Authors}

Rolf Hut - Water Resources Engineering, Delft University of Technology, $2628 \mathrm{CH}$ Delft, The Netherlands

Paul Vriend - Hydrology and Quantitative Water Management Group, Wageningen University, 6708 PB Wageningen, The Netherlands

Winnie de Winter - The North Sea Foundation, 3511 MJ Utrecht, The Netherlands

Marijke Boonstra - The North Sea Foundation, 3511 MJ Utrecht, The Netherlands

Tim H. M. van Emmerik - Hydrology and Quantitative Water Management Group, Wageningen University, 6708 PB Wageningen, The Netherlands

Complete contact information is available at: https://pubs.acs.org/10.1021/acs.est.0c08094

\section{Author Contributions}

C.T.J.R and T.H.M.v.E. conceived the study. C.T.J.R. and R.H. designed the statistical analysis procedure. P.V., M.B. and W.d.W helped to formulate the various hypotheses on macrolitter transport. C.T.J.R. executed the data analysis. All authors contributed to the writing and editing the of the manuscript.

\section{Funding}

This research was partly funded by the Dutch Ministry of Infrastructure and Water Management, Directorate-General for Public Works and Water Management (Rijkswaterstaat). The Clean River project is financed by Gieskes Strijbis Foundation, Adessium Foundation and the National Postcode Lottery Netherlands.

\section{Notes}

The authors declare no competing financial interest.

The preprocessed data and the scripts for the data analysis are available on https://zenodo.org/record/4650057.

\section{ACKNOWLEDGMENTS}

The authors thank all of the volunteers of the Clean River Project (Schone Rivieren) who collected and categorized the more than 150000 (and counting) litter items. They also thank the Schone Rivieren project partners: Instituut voor Natuureducatie (IVN) and the Plastic Soup Foundation. Credits for the picture used for the visual abstract: Clean Rivers Project.

\section{REFERENCES}

(1) Van Emmerik, T.; Schwarz, A. Plastic debris in rivers. WIREs Water 2020, 7, No. el398.

(2) McIlgorm, A.; Campbell, H. F.; Rule, M. J. The economic cost and control of marine debris damage in the Asia-Pacific region. Ocean Coastal Manage. 2011, 54, 643-651.

(3) Honingh, D.; et al. Urban River Water Level Increase Through Plastic Waste Accumulation at a Rack Structure. Front. Earth Sci. 2020, 8,28

(4) Lau, W. W. Y.; et al. Evaluating scenarios toward zero plastic pollution. Science 2020, 369, No. eaba9475.

(5) Lebreton, L. C. M.; Andrady, A. Future scenarios of global plastic waste generation and disposal. Palgrave Commun. 2019, 5, No. 6.

(6) Castro-Jiménez, J.; González-Fernández, D.; Fornier, M.; Schmidt, N.; Sempéré, R. Macro-litter in surface waters from the Rhone River: Plastic pollution and loading to the NW Mediterranean Sea. Mar. Pollut. Bull. 2019, 146, 60-66.

(7) van Emmerik, T.; et al. Riverbank macrolitter in the Dutch Rhine-Meuse delta. Environ. Res. Lett. 2020, 15, No. 104087.

(8) Martin, C.; et al. Exponential increase of plastic burial in mangrove sediments as a major plastic sink. Sci. Adv. 2020, 6, No. eaaz5593.

(9) Liro, M.; Emmerik, T.; van Wyżga, B.; Liro, J.; Mikuś, P. Macroplastic Storage and Remobilization in Rivers. Water 2020, 12, 2055

(10) Tramoy, R.; et al. Transfer dynamics of macroplastics in estuaries - New insights from the Seine estuary: Part 2. Short-term dynamics based on GPS-trackers. Mar. Pollut. Bull. 2020, 160, No. 111566

(11) Tramoy, R.; Gasperi, J.; Colasse, L.; Tassin, B. Transfer dynamic of macroplastics in estuaries - New insights from the Seine estuary: Part 1 . Long term dynamic based on date-prints on stranded debris. Mar. Pollut. Bull. 2020, 152, No. 110894.

(12) Ivar do Sul, J. A.; Costa, M. F.; Silva-Cavalcanti, J. S.; Araújo, M. C. B. Plastic debris retention and exportation by a mangrove forest patch. Mar. Pollut. Bull. 2014, 78, 252-257.

(13) Weideman, E. A.; Perold, V.; Ryan, P. G. Limited long-distance transport of plastic pollution by the Orange-Vaal River system, South Africa. Sci. Total Environ. 2020, 727, No. 138653.

(14) Lebreton, L. C. M.; et al. River plastic emissions to the world's oceans. Nat. Commun. 2017, 8, No. 15611.

(15) Meijer, L. J. J.; Van Emmerik, T.; Van der Ent, R.; Schmidt, C.; Lebreton, L. Over 1,000 rivers accountable for $80 \%$ of global riverine plastic emissions into the ocean. Submitt. Publ. Sci. Adv. 2019, 1-37.

(16) Vriend, P.; et al. Rapid Assessment of Floating Macroplastic Transport in the Rhine. Front. Mar. Sci. 2020, 7, 10.

(17) González-Fernández, D.; Hanke, G. Toward a harmonized approach for monitoring of riverine floating macro litter inputs to the marine environment. Front. Mar. Sci. 2017, 4, 86

(18) Kiessling, T.; et al. Plastic Pirates sample litter at rivers in Germany - Riverside litter and litter sources estimated by schoolchildren. Environ. Pollut. 2019, 245, 545-557.

(19) van Emmerik, T.; et al. A methodology to characterize riverine macroplastic emission into the ocean. Front. Mar. Sci. 2018, 5, 372.

(20) Vriend, P.; Roebroek, C.; van Emmerik, T. Same but different: A framework to design and compare riverbank plastic monitoring strategies. Front. Water 2020, 2, No. 563791.

(21) Owens, K. A.; Kamil, P. I. Adapting Coastal Collection Methods for River Assessment to Increase Data on Global Plastic Pollution: Examples From India and Indonesia. Front. Environ. Sci. 2020, 7, 208

(22) Hurley, R.; Woodward, J.; Rothwell, J. J. Microplastic contamination of river beds significantly reduced by catchment-wide flooding. Nat. Geosci. 2018, 11, 251-257.

(23) Roebroek, C. T. J.; et al. Plastic in global rivers: are floods making it worse? Environ. Res. Lett. 2021, 16, 025003.

(24) Pérez-Hernández, O.; Giesler, L. J.; Hilbe, J. M. A Negative Binomial Regression Model of the Observed Population Density of 
Heterodera glycines after Annual Corn Rotation in Nebraska. Plant Dis. 2019, 103, 3093-3100.

(25) Crotteau, J. S.; Ritchie, M. W.; Varner, J. M. A Mixed-Effects Heterogeneous Negative Binomial Model for Postfire Conifer Regeneration in Northeastern California, USA. For. Sci. 2014, 60, $275-287$.

(26) Boonstra, M.; Hougee, M. OSPAR Beach Litter Monitoring in the Netherlands 2013-2018: annual report - Publicatiedatabank IenW. http://publicaties.minienm.nl/documenten/ospar-beach-littermonitoring-in-the-netherlands-2013-2018-annual-report 2019. 